\title{
Time averaged properties along unstable periodic orbits and chaotic orbits in two map systems
}

\author{
Y. Saiki and M. Yamada \\ Research Institute for Mathematical Sciences, Kyoto University, 606-8502 Kyoto, Japan \\ Received: 18 February 2008 - Revised: 7 May 2008 - Accepted: 10 July 2008 - Published: 7 August 2008
}

\begin{abstract}
Unstable periodic orbit (UPO) recently has become a keyword in analyzing complex phenomena in geophysical fluid dynamics and space physics. In this paper, sets of UPOs in low dimensional maps are theoretically or systematically found, and time averaged properties along UPOs are studied, in relation to those of chaotic orbits.
\end{abstract}

\section{Introduction}

Chaotic dynamical systems often model complex phenomena in geophysics, space physics and fluid dynamics. Each chaotic system has an infinite number of unstable periodic orbits (UPOs), and the UPOs can play important roles in characterizing and analyzing the system. It is known that physical invariant measure of some chaotic systems can be captured by a weighted average of a series of many UPOs (Auerbach et al., 1987; Grebogi et al., 1988). In fact, physical invariant measure of the Hénon map is approximated well by a weighted average of a set of many UPOs with relatively low periods (Lai et al., 1997). Recently UPOs are numerically found in some high dimensional chaotic systems in geophysics and fluid turbulence for the purpose of capturing ordered structures in complex phenomena. Kazantsev studied UPOs in a barotropic ocean model and reported that only a few UPOs are sufficient to reconstruct some distribution functions of dynamical quantities and the attractor dimension (Kazantsev, 1998). Sensitivity of statistical averages of the solution to small external influences are also studied by using UPOs (Kazantsev, 2001). Rempel and Chian (2005) and Chian et al. (2006) discussed Alfvén intermittency in space plasma dynamics through UPOs. Kawahara and Kida (2001), Kato and Yamada (2003), van Veen et al. (2006) and Yamada

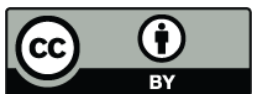

Correspondence to: Y. Saiki

(saiki@ kurims.kyoto-u.ac.jp) and Saiki (2007) extracted UPOs in fluid dynamics models and showed that they characterize turbulence properties. It is not easy to detect many UPOs from high dimensional chaotic systems even numerically, because in most cases they cannot be found by the forward time integration of the systems (Saiki, 2007). Actually small number of UPOs with low periods can be detected, but surprisingly chaotic behaviors in the above researches are captured well. For some Hamiltonian systems it is also reported that only one UPO is enough to approximate the Lyapunov exponent of the systems (Franzosi et al., 2005; Goto, 2007).

However, we do not have enough knowledge about statistical properties of UPOs, and we do not know why in some systems only a few UPOs with low periods out of an infinite number of UPOs can capture well statistical properties and ordered structures of complex behaviors. There are some researches related to this problem. Kawasaki and Sasa (2005) studied a simple model of chaotic dynamical systems with a large number of degrees of freedom, and found that there is an ensemble of UPOs with the special property that the expectation values of macroscopic quantities can be calculated using one UPO sampled from the ensemble. Invariant sets embedded in a chaotic attractor can generate time averages that differ from the average generated by typical orbits on the attractor. Motivated by two topics controlling chaos and riddled basins of attractions, Hunt and Ott (1996a, b) studied an optimal periodic orbit which yields the largest (optimal) value of a time average of a given smooth performance function of the system state. They obtained an implication that the optimal periodic orbit is typically a periodic orbit of low period, although they do not consider the relation of averaged statistical properties along UPOs and chaotic orbits. However, Yang et al. (2000) reported that the optimal UPO can be a periodic orbit of high period when the system is near crisis.

For developing the knowledge of chaotic analysis by UPOs, we study statistical properties of many UPOs and segments of chaotic orbits. This would also help us understand

Published by Copernicus Publications on behalf of the European Geosciences Union and the American Geophysical Union. 


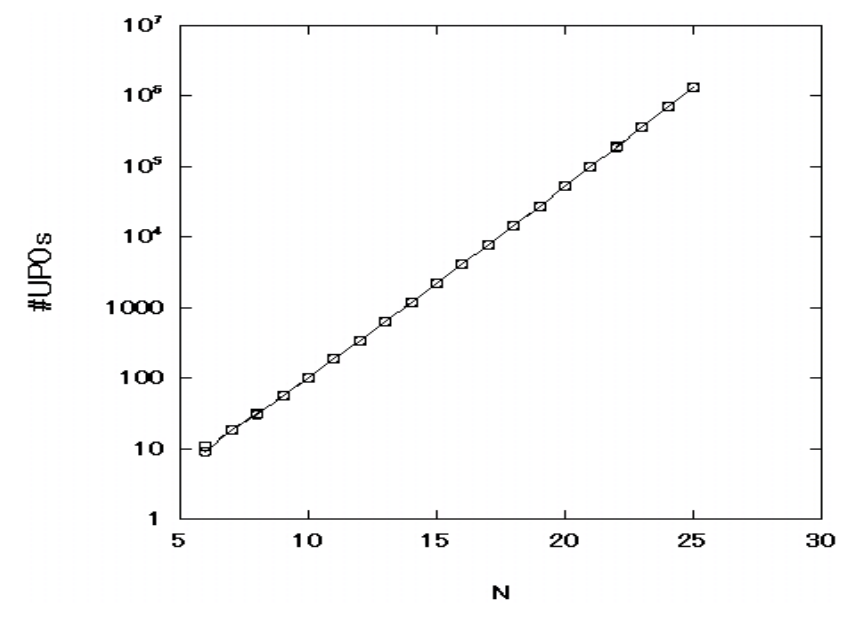

Fig. 1. The number of periodic orbits with periods $N(\mathrm{o})$ and $2^{N} / N(\square)$.

the difference between the maximal entropy measure and physical measure, which is not understood well. Although it is usually quite difficult to detect many UPOs from chaotic systems, there are a few exceptional chaotic systems in which UPOs can be analytically or systematically detected. Here, in this paper, we employ two low dimensional maps, logistic map and Hénon map, and study time averaged properties along UPOs and chaotic orbits directly. UPOs of the logistic map with a special parameter value are analytically calculated, and those of Hénon map are numerically identified by applying the method proposed in Biham and Wenzel (1989). In Sect. 2, statistical properties of UPOs and chaotic orbits of the logistic map are examined. In Sect. 3, a set of UPOs of the Hénon map is found, and statistical properties are studied comparing with those of segments of chaotic orbits. We summarize our results in the final section.

\section{Logistic map}

First we take the logistic map

$x_{n+1}=f\left(x_{n}\right)=4 x_{n}\left(1-x_{n}\right)$

on the interval $[0,1]$ as a simplest example of chaotic systems. Introducing a new variable $\theta$ as $x_{n}=\sin ^{2} \theta_{n}$, The logistic map is transformed to $\theta_{n+1} \equiv \pm 2 \theta_{n}(\bmod \pi)$, which leads to $\theta_{n} \equiv \pm 2^{n-1} \theta_{1}(\bmod \pi)$. The $N$-periodic solutions $x_{N+1}=x_{1}$ thus correspond to $\theta_{N+1} \equiv \pm \theta_{1}(\bmod \pi)$, and are realized by the initial points $\theta_{1}^{ \pm}=k \pi /\left(2^{N} \pm 1\right)$, where $0 \leq k \leq 2^{N-1}$ for $\theta_{1}^{+}$ and $1 \leq k \leq 2^{N-1}-1$ for $\theta_{1}^{-}$. Note that the total number of periodic points is $2^{N}$.

In the following in this paper, we define the periodic orbits with period $N$ as those that have period $N$ but does not have shorter period than $N$. In this sense, the number of periodic orbits of period $N$ is well estimated by $2^{N} / N$ (Fig. 1), because the number of periodic orbits with period $\leq N / 2$ has an upper bound $\sum_{i=1}^{N / 2} 2^{i} / i \leq 2^{N / 2+1}$ which is negligible compared to $2^{N}$ for large $N$. Let us calculate the orbital mean $y=M_{N}\left(x_{1}\right)$ of $x$ along the individual periodic orbit of period $N$, defined as $M_{N}\left(x_{1}\right)=\sum_{n=1}^{N} x_{n} / N$ where $\left\{x_{1}, x_{2}, \cdots, x_{N}\right\}$ is a periodic orbit with period $N$. According to the distribution of the initial value of $x_{1}, y=M_{N}\left(x_{1}\right)$ also has a distribution $P_{N}(y)$ on $[0,1]$.

Figure 2 (left) shows the numerically obtained density distribution $P_{N}(y)$ for $N=22,23,24,25$, where we can see a little but clear asymmetry with respect to $y=1 / 2$, and see that the density distribution is nearly the same for these values of $N$. We note that in our calculation we obtain the periodic orbits analytically by using $\theta_{n+1} \equiv 2 \theta_{n}$, while a numerical calculation of the periodic orbits, without using the analytical results, is not available because the product of $\left|d f^{(N)} / d x\right|\left(\sim 2^{N}\right)$ and the round-off error $\left(10^{-15}\right.$ in double precision) is comparable to the minimum distance between adjacent periodic points for $N \geq 17$. We also show in Fig. 2 (right) the density distribution function of the orbital mean $M_{N}$ along a chaotic orbit, where we decompose a chaotic orbit into segments with length $N$, and numerically calculate the mean of $x$ for each of these segments. The number of segments with length $N$ is taken to be the same as those of periodic orbits with period $N$. We see that the density distributions of $y=M_{N}$ almost coincide with each other.

The observation is supported by the following analytical discussion. Noting that the physical invariant measure is given by $P_{\text {inv }}(x) d x=d x /(\pi \sqrt{x(1-x)})=2 d \theta / \pi \quad\left(x=\sin ^{2} \theta\right), \quad$ we may construct two probabilistic models for $P_{N}(y)$. In the first model, we boldly assume that the points $x_{1}, x_{2}, \cdots, x_{N}$ are chosen independently of each other in a random way according to the invariant measure $P_{\mathrm{inv}}(x)$. In the second model, we assume that only the initial point $x_{1}$ is randomly chosen according to $P_{\text {inv }}(x)$ and the subsequent points $x_{2}, \cdots, x_{N}$ are determined by $x_{n+1}=f\left(x_{n}\right)$. Interestingly, both of these models give the same values of the mean $m_{N}$ and the variance $\sigma_{N}^{2}$ of $P_{N}(y)$, as $m_{N}=1 / 2, \sigma_{N}^{2}=1 /(8 N)$. Actually, the standard deviation of $P_{N}(y)$ for the periodic orbits, the segments of a chaotic orbit and those obtained by the probabilistic models agree fairly well with each other for larger $N$ (Fig. 3). However, the first model is not satisfactory, because it gives the vanishing third order cumulant which does not agree with the numerical result where the asymmetry of $P_{N}(y)$ yields a nonzero value for the third order cumulant. On the other hand, the second model gives the third order cumulant equal to $-3(N-1) /\left(32 N^{3}\right)$, which agrees well with the numerical result (Fig. 4). This agreement is also supported by an observation that the physical invariant measure $P(x)_{\text {inv }} d x$ of $f(x)$ is also the physical invariant measure of $f^{N}(x)$. Therefore, hereafter, we take the second model as a statistical model for the $M_{N}$. 

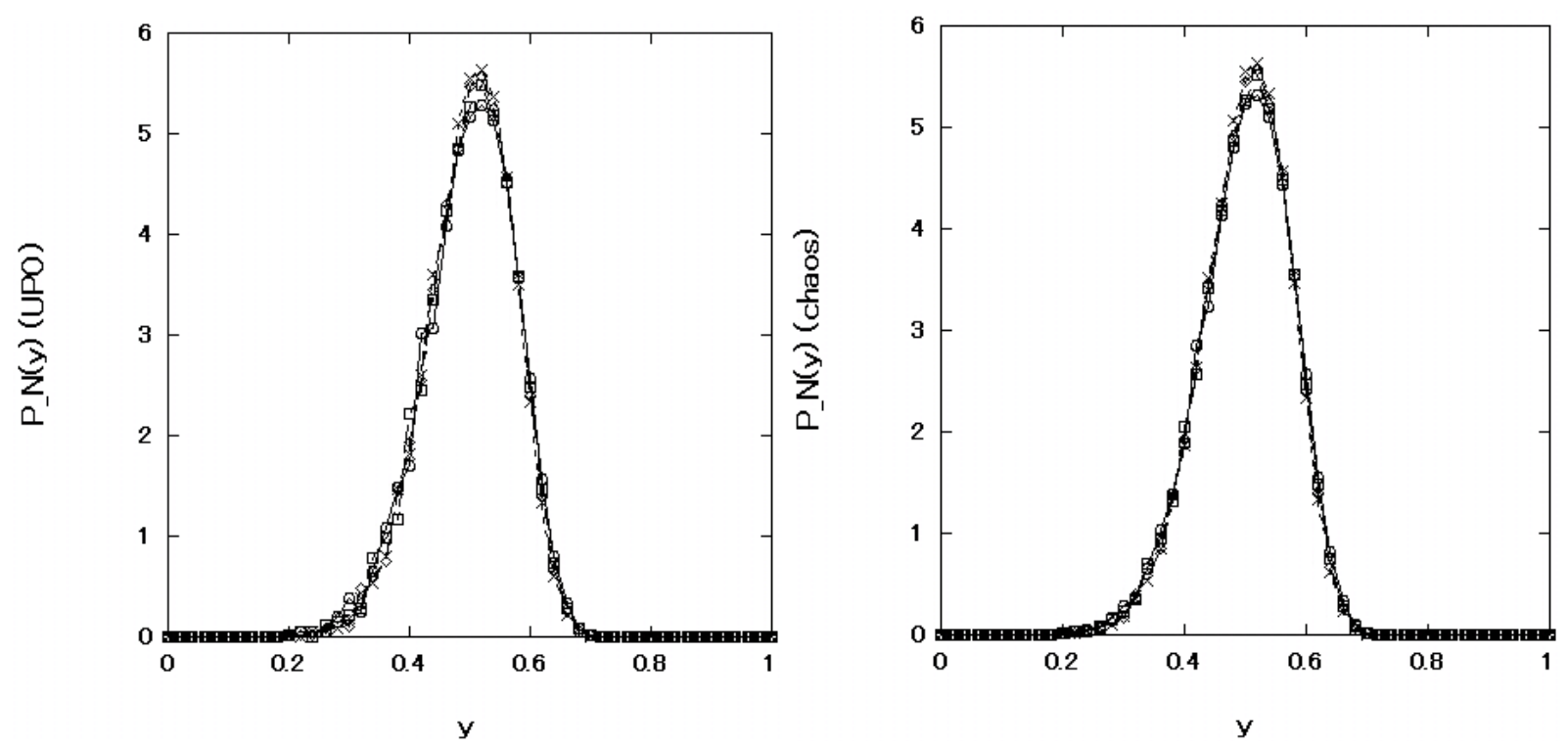

Fig. 2. Density distribution $P_{N}(y)$ for periodic orbits (left), and for chaotic orbits (right) with lengths $N=22(\circ), 23(\square), 24(\diamond), 25(\times)$.

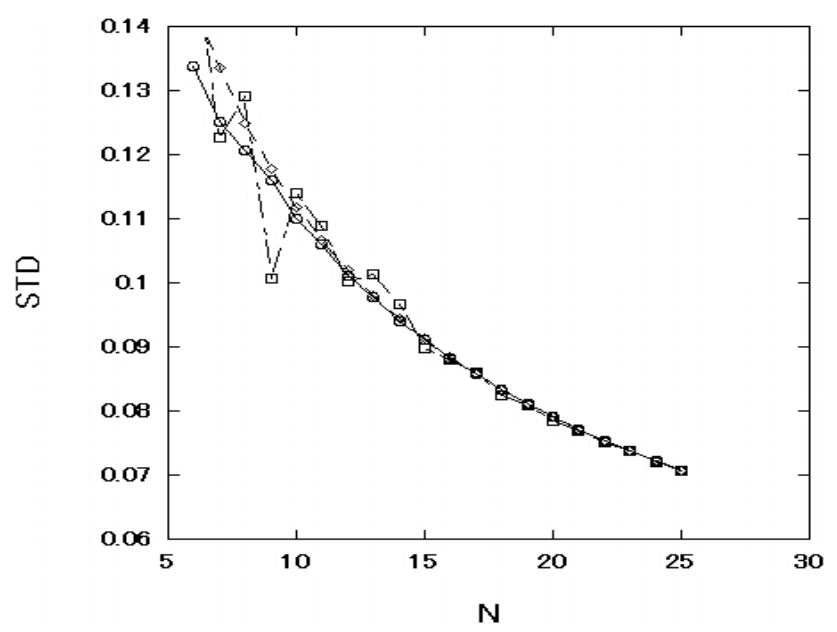

Fig. 3. Standard deviation of the density distribution $P_{N}(y)$ for periodic orbits (o), segments of a chaotic orbit $(\square)$, and segments obtained by probabilistic models $(\diamond)$.

We now calculate the characteristic function $\Psi_{N}(\lambda)$ of $P_{N}(y)$ for large $N$, where $y=M_{N}(x)=\sum_{n=1}^{N} f^{n}(x)$ and the probability density function of $x$ is $P_{\text {inv }}(x)$,

$$
\begin{aligned}
\Psi_{N}(\lambda) & =\int_{0}^{1} \exp \left[i \lambda M_{N}(x)\right] P_{\text {inv }}(x) d x \\
& =\frac{2}{\pi} \int_{0}^{\pi / 2} \exp \left[i \lambda M_{N}(x)\right] d \theta
\end{aligned}
$$

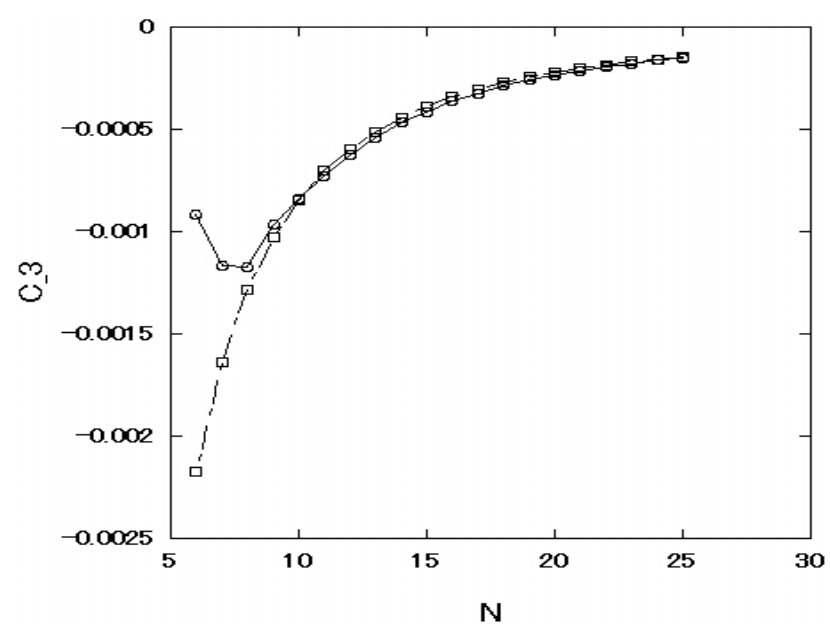

Fig. 4. The third order cumulant of the density distribution $P_{N}(y)$ for periodic orbits (०), and segments of a chaotic orbit ( $\square$ ).

$$
\begin{aligned}
& \sim \frac{2}{\pi} \frac{1}{2}\left[\sum_{k=1}^{2^{N-1}} \exp \left[i \lambda M_{N}\left(\sin ^{2} \theta_{k}^{+}\right)\right] \frac{\pi}{2^{N}+1}\right. \\
& \left.\quad+\sum_{k=1}^{2^{N-1}-1} \exp \left[i \lambda M_{N}\left(\sin ^{2} \theta_{k}^{-}\right)\right] \frac{\pi}{2^{N}-1}\right] \\
& \sim \frac{1}{2^{N}} \sum^{\prime} \exp \left[i \lambda M_{N}\left(\sin ^{2} \theta_{k}^{ \pm}\right)\right] .
\end{aligned}
$$

Here we approximate the integral by the mean of finite sums of two kinds of mensurations by division, and $\sum^{\prime}$ denotes the summation over all the periodic points. As stated before, 


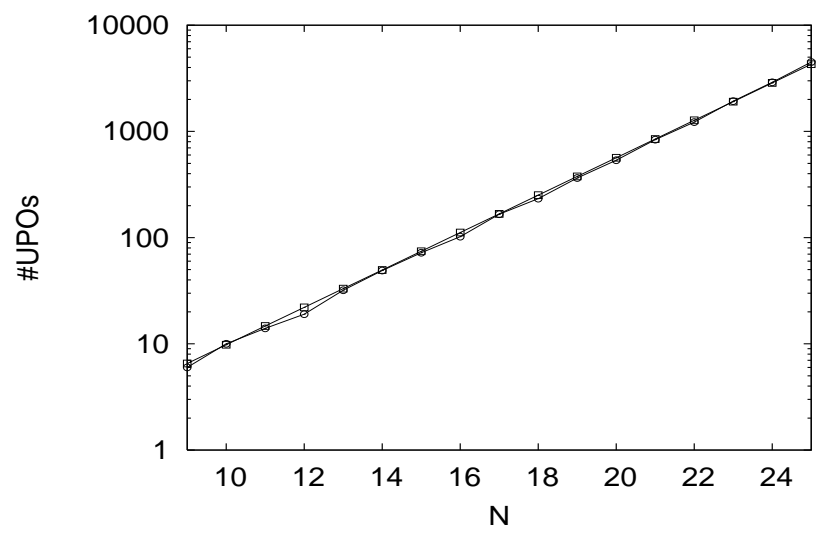

Fig. 5. The number of detected periodic orbits with periods $N$ (०) and $0.17 \cdot 1.5^{N}(\square)$.

the number of periodic orbits with period $\leq N / 2$ is negligible compared to that with period $N$. Therefore, $\sum^{\prime}$ is approximated by $N \sum_{\mathrm{UPO}(N)}$ where the latter means the sum over all the periodic orbits with period $N$, and we have taken into account the fact that a periodic orbit with period $N$ has $N$ periodic points. We then have

$$
\begin{aligned}
\Psi(\lambda) & =\frac{1}{2^{N} / N} \sum_{\operatorname{UPO}(N)} \exp \left[i \lambda M_{N}\right] \\
& \sim<\exp \left[i \lambda M_{N}\right]>\operatorname{UPO}(N)
\end{aligned}
$$

where $<\cdot>_{\operatorname{UPO}(N)}$ means the average over the periodic orbits with period $N$. Thus we conclude that for large $N$, the density distribution of $M_{N}$ for a chaotic orbit, agrees with the density distribution of $M_{N}$ over the periodic orbits with period $N$.

This conclusion implies that, at least with respect to the distribution $P_{N}$ of the orbital mean $M_{N}$ as $N \rightarrow \infty$, the UPOs give statistically equal results to that of chaotic segments in the logistic map. In other words, if we analyze time averaged properties of a variable of the logistic map, we should take periodic orbits with longer periods to obtain a better convergence of the result. In this respect, therefore, the logistic map appears different from some high dimensional systems in geophysics and fluid dynamics, where only a few UPOs with rather short periods are known to give remarkably good approximations to statistical properties of chaotic orbits (Kazantsev, 1998, 2001; Kawahara and Kida, 2001; Kato and Yamada, 2003; van Veen et al., 2006; Yamada and Saiki, 2007).

\section{Hénon map}

Hénon map (Hénon (1976)) is a two-dimensional map on $\mathbf{R}^{2}$, which is described by

$x_{n+1}=a-x_{n}^{2}+y_{n}, y_{n+1}=b x_{n}$, where the parameters $a, b(\in \mathbf{R})$ are constants. This is a diffeomorphism if $b \neq 0$, and the Jacobian determinant of the system is $-b$. Hénon map is sometimes described as $\left(x_{n+1}, y_{n+1}\right)=\left(a-x_{n}^{2}+b y_{n}, x_{n}\right),\left(b y_{n}+1-a x_{n}^{2}, x_{n}\right)$ by the Affine transformation of the above system. Hénon map is the only one diffeomorphism on $\mathbf{R}^{2}$ described by a polynomial of order 2 and the inverse of which is also written by a polynomial. An orbit of the Hénon map is essentially determined as a sequence on $\mathbf{R}$ by $x_{n+1}=a-x_{n}^{2}+b x_{n-1}$ and $\left\{y_{n}\right\}$ is determined by $\left\{x_{n}\right\}$ as $y_{n+1}=b x_{n}$.

UPOs cannot be extracted by the forward time iteration of the original system because of their instabilities. However, in the case of the Hénon map, there is a special technique for finding UPOs, which is proposed in Biham and Wenzel (1989). In this method UPOs with period $N$ are identified by the attracting fixed points of the corresponding $\mathrm{N}$-dimensional ordinary differential equation systems (ODE systems):

$\frac{d x_{n}}{d t}=\sigma_{n}\left(-b x_{n-1}-x_{n+1}+a-x_{n}^{2}\right),(n=0, \cdots . N-1)$

where $x_{-1}=x_{N-1}, x_{N}=x_{0}$, and $\sigma_{n}= \pm 1(n=0, \cdots, N-1)$. $2^{N}$ sorts of ODE systems are obtained by choosing a set of $\left(\sigma_{0}, \cdots, \sigma_{N-1}\right)$. Attracting fixed points $\left(x_{0}^{*}, \cdots, x_{N-1}^{*}\right)$ are found from these systems. It is known that each fixed point corresponds to one UPO of the Hénon map (Biham and Wenzel, 1989). The method is considered to identify all the periodic orbits with period $N$ of the Hénon map in some parameter values by obtaining all the attracting fixed points of $2^{N}$ sorts of $\left(\sigma_{0}, \cdots, \sigma_{N-1}\right)$ (Grassberger et al., 1989; Davis et al., 1991; Sterling and Meiss, 1998), although there is still no mathematical proof for it.

In this paper by employing the method we obtain more than 10000 periodic orbits with periods $N \leq 25$ of the Hénon map with the parameter values set as $a=1.4, b=0.3$. The number of UPOs with period $N$ shown in Fig. 5 grows exponentially (cf. Bowen, 1970), which indicates the topological entropy of the system to be about $\log (1.5)$. To compare statistical properties of UPOs with those of chaotic orbits we consider a set of segments of chaotic orbits (chaotic segments) with length $N$. Initial points $x_{1}$ of the chaotic segments are chosen according to the physical invariant measure which is numerically obtained. We focus on the time averaged value of $x\left(M_{N}\left(x_{1}\right)\right)$ of a segment $\left\{x_{n}\right\}_{n=1, \cdots, N}$ with time length $N$, which is calculated by $M_{N}\left(x_{1}\right)=\sum_{n=1}^{N} x_{n} / N$. The density distributions $P_{N}(z)$ of $z=M_{N}\left(x_{1}\right)$ are shown in Fig. 6 for all the detected UPOs and $10^{5}$ chaotic segments with length $N$. We can find that the distributions of $M_{N}\left(x_{1}\right)$ with time lengths $N$ of UPOs are significantly different from those of chaotic segments with the same time lengths. The observation is confirmed in Fig. 7 which shows the averages(upper) and standard deviations(lower) of the density distributions $P_{N}(z)$ of $z=M_{N}\left(x_{1}\right)$ of UPOs and chaotic segments with time lengths $N$. From Fig. 7 we can see that distributions $P_{N}(z)$ of $z=M_{N}\left(x_{1}\right)$ along UPOs and chaotic segments with 


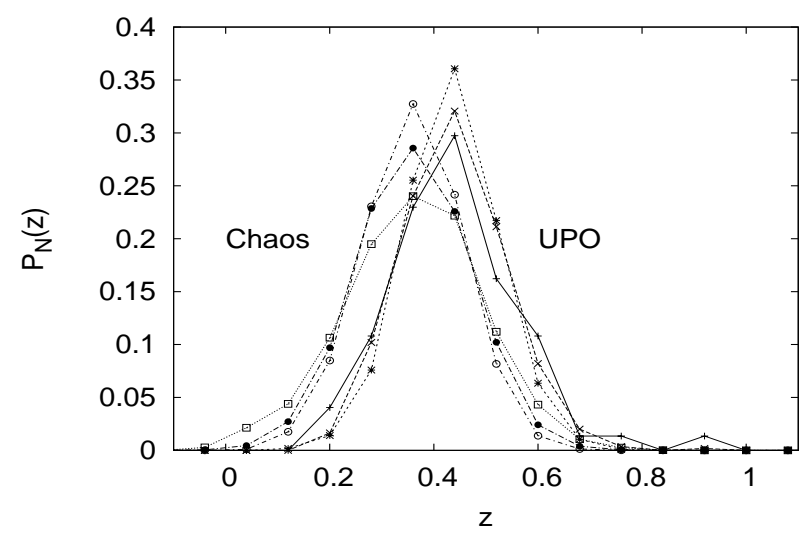

Fig. 6. Density distribution $P_{N}(z)$ of time averaged value of $x$ $\left(z=M_{N}\left(x_{1}\right)\right)$ along periodic orbits with periods $N(=15(+), 20(\times)$, $25(*))$ and chaotic segments with lengths $N(=15(\square), 20(\bullet), 25$ (०)).

time lengths $N$ seem to converge to the different distributions which have different mean values as $N$ increases. Our results suggest that in the case of the Hénon map, time averaged properties of chaotic orbits cannot be captured by using a few UPOs.

\section{Conclusions}

In this paper we study statistical properties of time averaged values of a dependent variable along unstable periodic orbits (UPOs) and chaotic segments of the logistic map and the Hénon map. UPOs of these two maps with the parameter values employed in this paper are found by theoretical or systematical numerical calculations. In the case of the logistic map, we can find that averaged values of a variable along chaotic orbits and UPOs have almost the same properties. On the other hand, in the Hénon map, the distribution of averaged values of a dependent variable numerically obtained along the UPOs does not appear to coincide with that along the segments of a chaotic orbit. The result in the Hénon map may seem contradictory to the findings of Lai et al. (1997), which shows that the physical invariant measure is approximately obtained by a series of UPOs with a weight proportional to the reciprocal of the largest Floquet exponent. However, the distributions we considered here are obtained by the use of the UPOs with no weight employed, and thus are related to the measure of maximal entropy. It is still an open problem whether these averaged values converge to a single value, because the above results have been obtained only by numerical calculations. However, it is impressive that neither of these typical chaotic map systems of low dimensions shows a straightforward support for the recent UPO applications in geophysics and fluid dynamics, where averaged values along only a few UPOs are in good agreement with
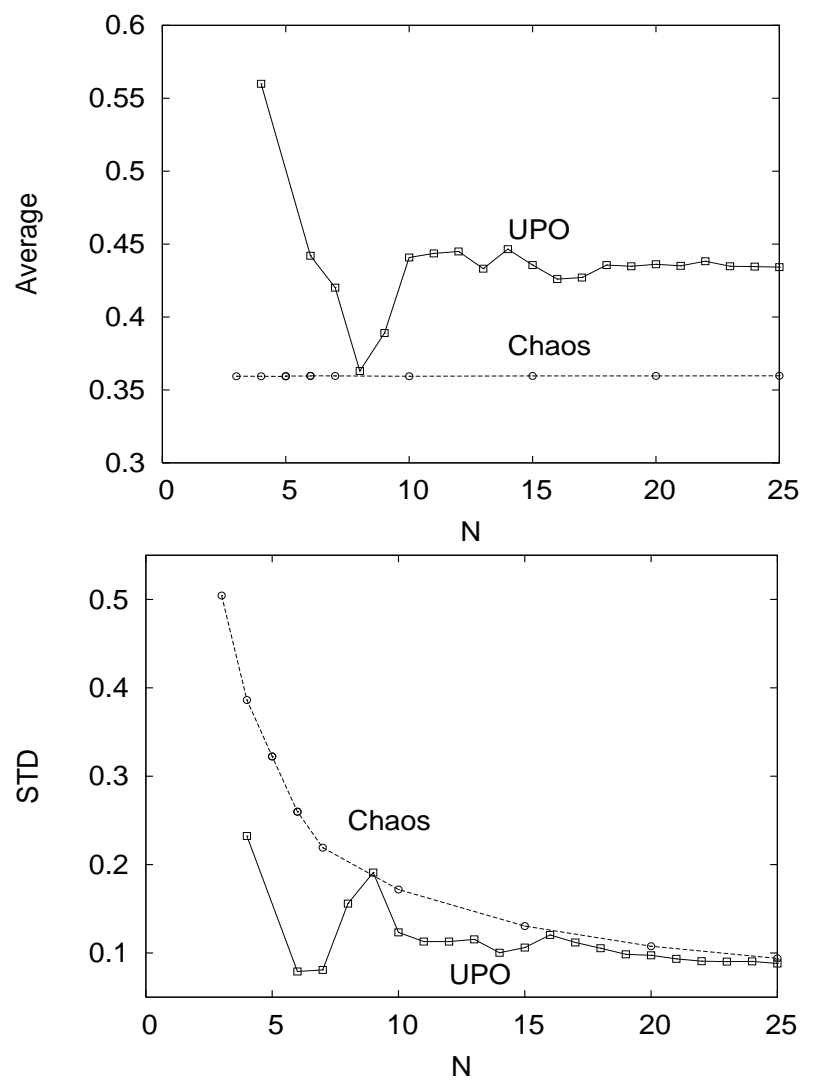

Fig. 7. Average(upper) and standard deviation(STD)(lower) of the density distribution $P_{N}(z)$ of time averaged value of $x\left(z=M_{N}\left(x_{1}\right)\right)$ along periodic orbits $(\square)$ and chaotic segments (o) with timelengths $N$.

averaged values along a chaotic orbit. Research in this direction is now on progress for systems of differential equations and will be reported elsewhere.

Acknowledgements. The authors are very thankful to the anonymous referees for their insightful comments. This work is partially supported by the Grant-in-Aids 16340023, 19654019 and 194048.

Edited by: A. C. L. Chian

Reviewed by: three anonymous referees

\section{References}

Auerbach, D., Cvitanović, P., Eckmann, J. P., Gunaratne, G., and Procaccia, I.: Exploring chaotic motion through periodic orbits, Phys. Rev. Lett., 58, 2387-2389, 1987.

Biham, O. and Wenzel, W.: Characterization of unstable periodic orbits in chaotic attractors and repellers, Phys. Rev. Lett., 63, 819-822, 1989.

Bowen, R.: Topological entropy and AxiomA, Global Analysis (Berkeley, CA, 1968) Proc. Sympos. Pure Math., 14, Amererican Mathathematical Society, Providence, RI, 23-41, 1970. 
Chian, A. C. L., Kamide, Y., Rempel, E. L., and Santana, W. M.: On the chaotic nature of solar-terrestrial environment: interplanetary Alfvén intermittency, J. Geophys. Res., 111(10), A07S03, doi:10.1029/2005JA011396, 2006.

Davis, M. J., MacKay, R. S., and Sannami, A.: Markov shifts in the Hénon family, Physica D, 52, 171-178, 1991.

Franzosi, R., Poggi, P., and Cerruti-Sola, M.: Lyapunov exponents from unstable periodic orbits, Phys. Rev. E, 71, 36218, doi:10.1103/PhysRevE.71.036218, 2005.

Grassberger, P., Kantz, H., Moenig, U.: On the symbolic dynamics of the Hénon map, J. Phys. A: Math. Gen., 22, 5217-5230, 1989.

Goto, S.: From an Unstable Periodic Orbit to the Lyapunov Exponent and a Macroscopic Variable in a Hamiltonian Lattice, Progress of Theoretical Physics, 118(1), 25-34, 2007.

Grebogi, C., Ott, E., and Yorke, J. A.: Unstable periodic orbits and the dimensions of multifractal chaotic attractors, Phys. Rev. A 37, 1711-1724, doi:10.1103/PhysRevA.37.1711, 1988.

Hénon, M.: A two-dimensional mapping with a strange attractor, Commun. Math. Phys., 50, 69-77, 1976.

Hunt, B. R. and Ott, E.: Optimal Periodic Orbits of Chaotic Systems, Phys. Rev. Lett., 76(13), 2254-2257, 1996a.

Hunt, B. R. and Ott, E.: Optimal Periodic Orbits of Chaotic Systems occur at low period, Phys. Rev. E, 54, 328-337, 1996b.

Kato, S. and Yamada, M.: Unstable periodic solutions embedded in a shell model turbulence, Phys. Rev. E, 68(2), R25 302-25 305, 2003.

Kawahara, G. and Kida, S.: Periodic motion embedded in plane Couette turbulence: regeneration cycle and burst, J. Fluid Mech., 449, 291-300, 2001.

Kawasaki, M. and Sasa, S.: Statistics of unstable periodic orbits of a chaotic dynamical system with a large number of degrees of freedom Phys. Rev. E, 72(3), 037202, doi:10.1103/PhysRevE.72.037202, 2005.
Kazantsev, E.: Unstable periodic orbits and attractor of the barotropic ocean model, Nonlin. Processes Geophys., 5, 193208, 1998, http://www.nonlin-processes-geophys.net/5/193/1998/.

Kazantsev, E.: Sensitivity of the attractor of the barotropic ocean model to external influences: approach by unstable periodic orbits, Nonlin. Processes Geophys., 8, 281-300, 2001, http://www.nonlin-processes-geophys.net/8/281/2001/.

Lai, Y.-C., Nagai, Y., and Grebogi, C.: Characterization of the Natural Measure by Unstable Periodic Orbits in Chaotic Attractors, Phys. Rev. Lett., 79, 649-652, 1997.

Rempel, E. L. and Chian, A. C.-L.: Space plasma dynamics: Alfven intermittent Chaos, Adv. Space Res., 35, 951-960, 2005.

Saiki, Y.: Numerical detection of unstable periodic orbits in continuous-time dynamical systems with chaotic behaviors, Nonlin. Processes Geophys., 14, 615-620, 2007, http://www.nonlin-processes-geophys.net/14/615/2007/.

Sterling, D. and Meiss, J. D.: Computing periodic orbits using the anti-integrable limit, Phys. Lett. A, 241, 46-52, 1998.

van Veen, L., Kida, S., and Kawahara, G.: Periodic motion representing isotropic turbulence, Fluid Dynam. Res., 38(1), 19-46, 2006.

Yamada, M. and Saiki, Y.: Chaotic properties of a fully developed model turbulence, Nonlin. Processes Geophys., 14, 631640, 2007, http://www.nonlin-processes-geophys.net/14/631/2007/.

Yang, T.-H., Hunt, B., and Ott, E.: Optimal periodic orbits for continuous time systems, Phys. Rev. E., 62, 1950-1959, 2000. 